\title{
Use of the cryostat section in electron microscopy
}

\author{
P NORRIS, DWR GRIFFITHS
}

From the Department of Pathology, Weston Park Hospital, Whitham Road, Sheffield S10 2SJ

SUMMARY A method using the cryostat section as a source of material from which relevant areas for ultrastructural examination can be positively located and quickly processed is described. Preservation of the tissue does not appear to be unduly affected by freezing and subsequent cryostat sectioning.

The rapid processing technique as described by Rowden and Lewis ${ }^{1}$ could be considered a milestone in the processing of clinical material for electron microscopy. The method described by these authors reduced the total processing time to three hours instead of the more conventional three to five days. ${ }^{2}$ In the technique described below the processing time is reduced again by half by using the cryostat section, which unlike the standard electron microscopy tissue block, permits precise localisation of selected areas.

The method retains the simplicity and precise localisation of the paraffin wax section technique as described by Gonzalez-Angulo et al, ${ }^{3}$ together with the preservation achieved by prompt glutaraldehyde fixation. ${ }^{4}$

Although relatively short processing techniques are available ${ }^{5-7}$ this method will allow "late" specimens to be processed to Araldite resin within 30 min, thus encouraging a more flexible and versatile electron microscopy service.

\section{Material and methods}

Transverse slices of mouse kidney ( $3 \mathrm{~mm}$ thick) were frozen onto an OCT (manufactured by Labtek division of Miles laboratories) coated cryostat chuck using a commercial dichlorodifluoromethane spray (Cryojet manufactured by Raymond A Lamb, London). Serial sections (10 $\mu$ m thick) were cut.

Light microscopic examination was performed on alternate sections after conventional air drying, formalin fixation and haematoxylin and eosin staining procedures adopted for urgent biopsy work (Fig. 1).

The remaining sections for electron microscopy were fixed immediately, without air drying, in phosphate-buffered glutaraldehyde at $4^{\circ} \mathrm{C}$ for one minute prior to processing. Specific localisation could be obtained by examination of the unstained

Accepted for publication 30 March 1983 cryostat section following fixation using phase contrast or interference microscopy.

Areas of interest were located in the sections stained for light microscopy and the corresponding areas relocated on the sections for electron microscopy by scoring the underside of the slides. Areas of interest on sections examined by phase contrast microscopy could be similarly identified. These sections were then processed in the following manner:

1 Rinse in $0 \cdot 1 \mathrm{M}$ sucrose phosphate buffer

$3 \times 10 \mathrm{~s}$

2 Osmicate in $2 \%$ unbuffered osmium tetroxide

3 Rinse in $99 \%$ alcohol $10 \mathrm{~s}$

4 Stain with saturated alcoholic uranyl acetate

5 Rinse in $99 \%$ alcohol

6 Rinse in $100 \%$ alcohol

7 Epoxy propane

8 Araldite resin:epoxy propane 50:50

9 Araldite resin

$5 \mathrm{~min}$

$5 \mathrm{~min}$

$3 \times 10 \mathrm{~s}$

$3 \times 10 \mathrm{~s}$

$6 \times 10 \mathrm{~s}$

$2 \times 3 \mathrm{~min}$

$5 \mathrm{~min}$

Surplus resin was removed from around the edge of the selected area and the glass slide was then inverted (section downwards) on top of silicon rubber moulds (TAAB Enterprises, Reading) previously filled with fresh Araldite resin. The resin was cured by polymerising at $100^{\circ} \mathrm{C}$ for one hour. The block with its attached slide was released from the embedding mould. The glass slide was removed by inserting a razor blade under the edge of the still warm block and detaching the block with the accompanying section from the glass slide (Sokol et $\left.a l^{8}\right)$. The blocks were rapidly cooled by placing into a $-20^{\circ} \mathrm{C}$ deep freeze for a few minutes. Localisation was re-established by examination of the block face under a light microscope (Fig. 2), alternatively this could be achieved by cutting $1 \mu \mathrm{m}$ sections and staining with $1 \%$ toluidine blue. After appropriate orientation, $50 \mathrm{~nm}$ sections were then cut on a Reichert OM U2 ultramicrotome, floated 903 
Fig. 1 Haematoxylin and eosin stained cryostat section featuring a single'mouse renal glomerulus. $\times 1100$
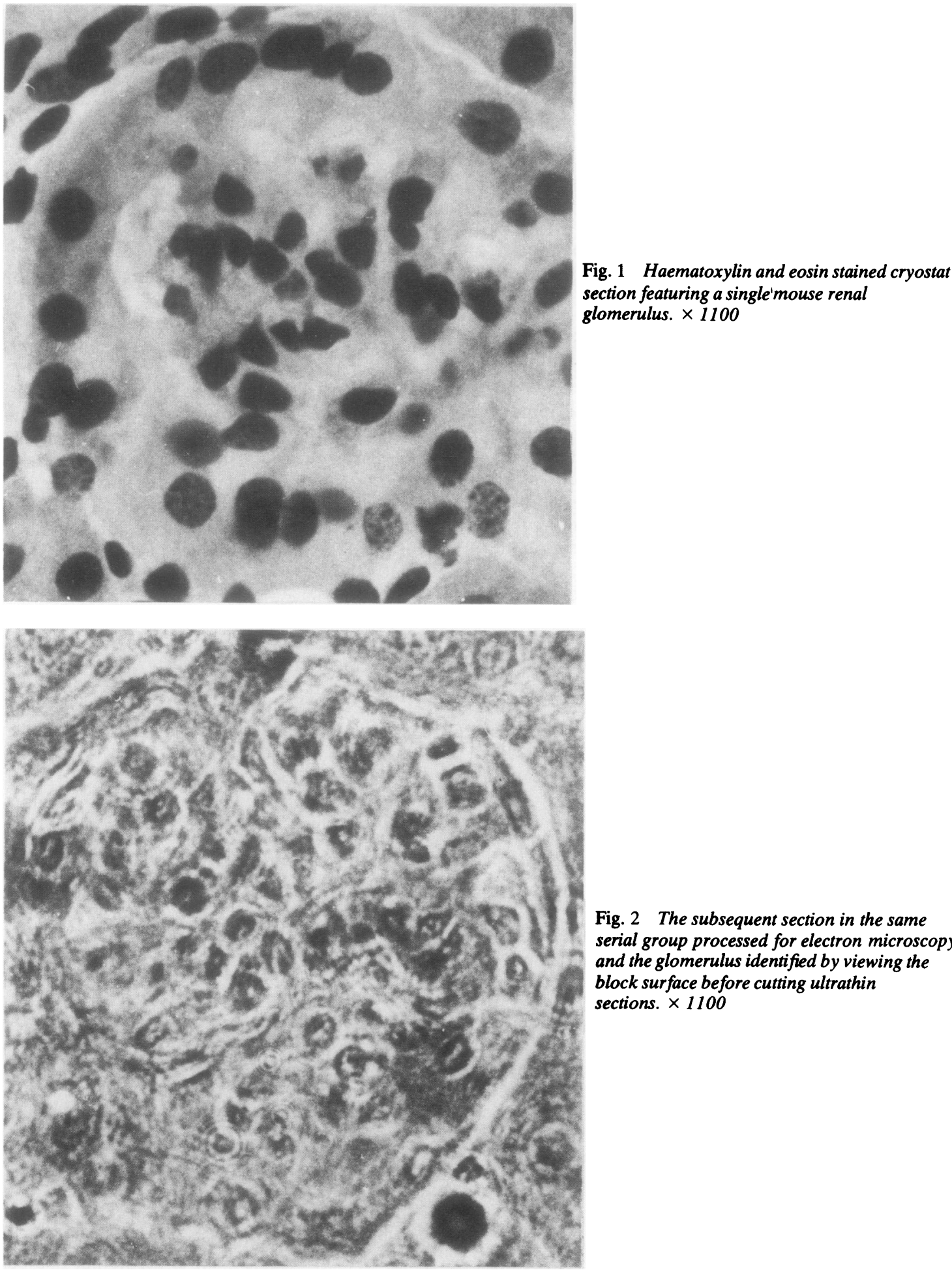

Fig. 2 The subsequent section in the same serial group processed for electron microscopy and the glomerulus identified by viewing the block surface before cutting ultrathin sections. $\times 1100$ 


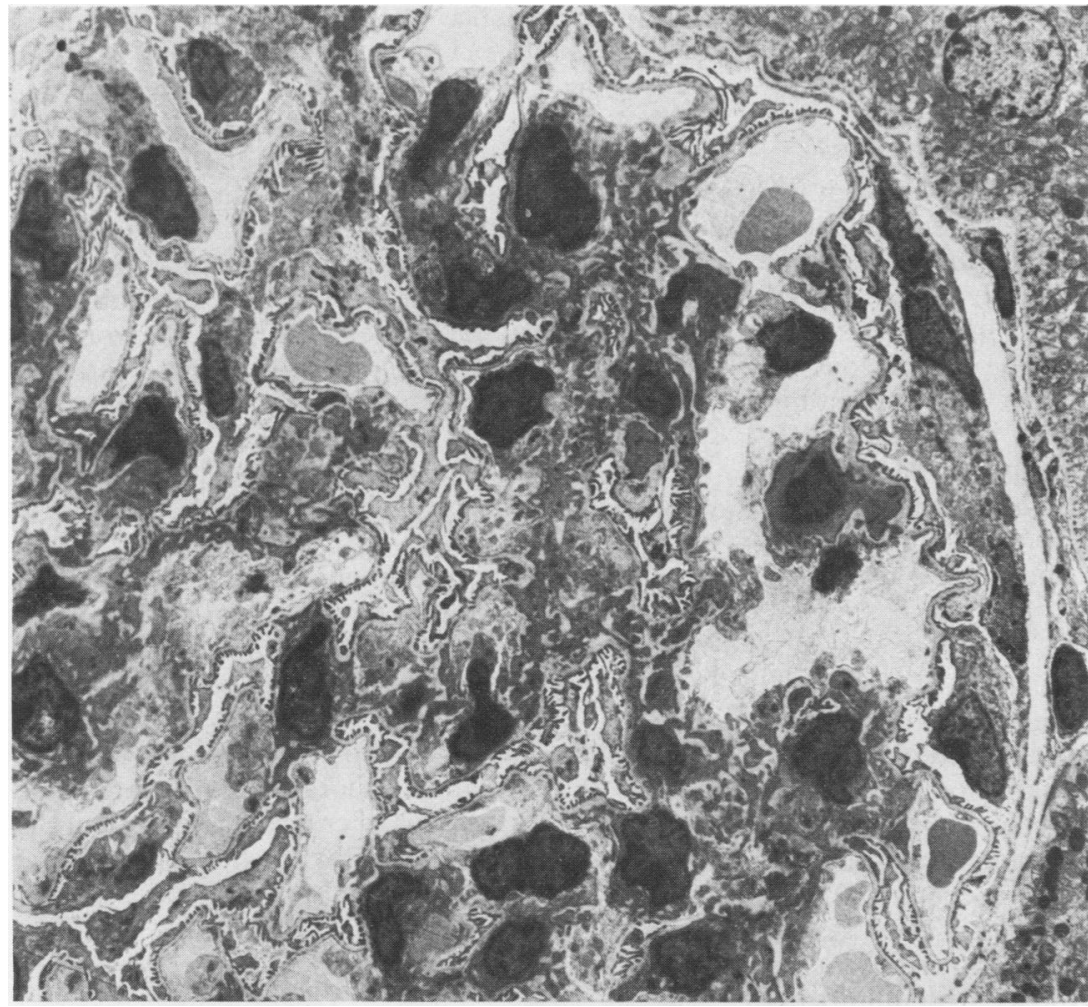

Fig. 3 Low powered electron micrograph of a $50 \mathrm{~nm}$ thick section demonstrating the same renal glomerulus. $\times 2000$

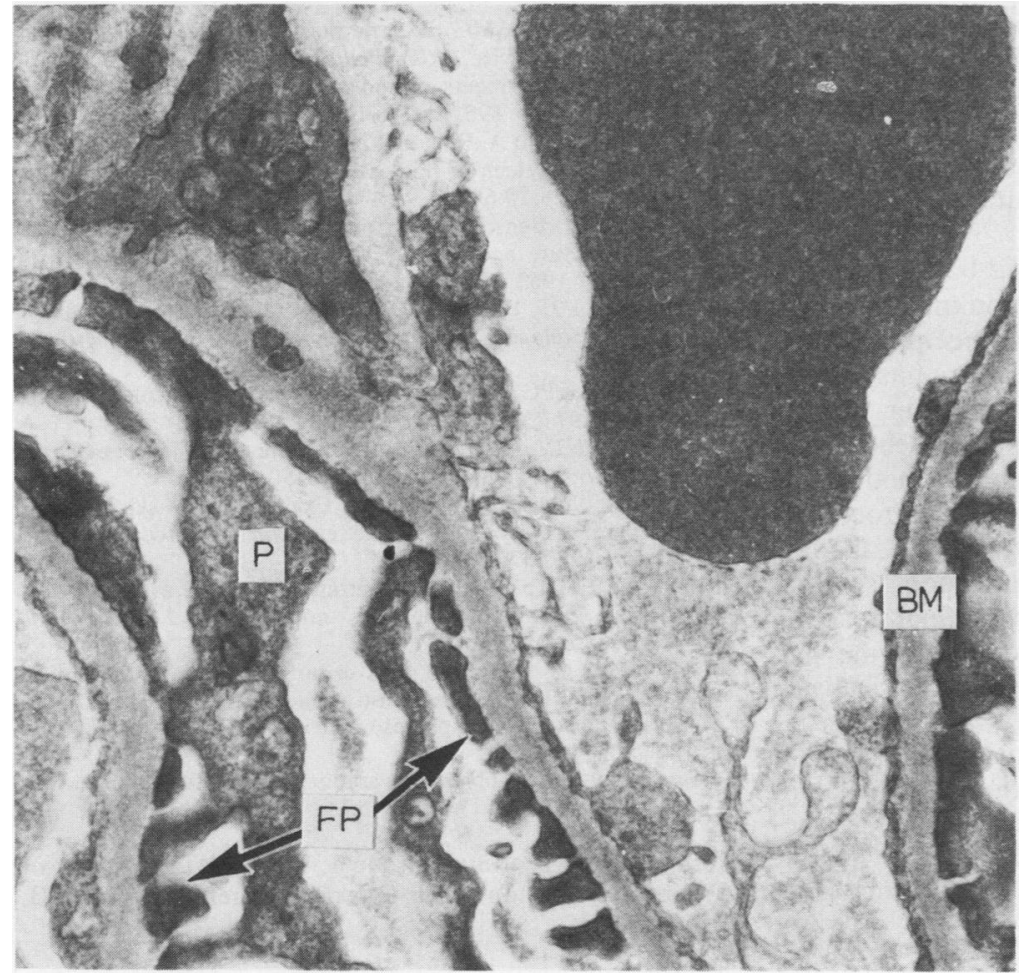

Fig. 4 Electron micrograph of an area shown in Fig. 3. BM = basement membrane; $P=$ podocyte; $F P=$ foot processes (arrows) $\times 20000$ 
out onto copper grids, stained with lead citrate ${ }^{9}$ and examined with a Philips $400 \mathrm{~T}$ electron microscope (Fig. 3, 4).

\section{Results}

See Figs. 1-4. On examination it appeared that the specimens processed in the manner described showed no ultrastructural damage and ice crystal artefact was not observed. In view of the limited amount of material available $(10 \mu \mathrm{m})$, orientation for electron microscopy should be carried out by visualising the block face rather than by cutting semi-thin sections for toluidine blue staining. It should be noted that when comparing the stained cryostat section examined at light microscopy with the corresponding area on the block face or the toluidine blue stained section, the image is reversed.

\section{Discussion}

From the results obtained, it would seem that ultrastructural damage is negligible when processing cryostat sectioned material for electron microscopy studies. By using the cryostat section as a source of tissue for electron microscopy, good localisation can be achieved by comparing these sections with the serial sections cut and stained for light microscopy.

If phase contrast or interference microscopy has been used on the glutaraldehyde fixed but unprocessed cryostat section, specific localisation can be achieved equal to that attained by the removal of selected areas from paraffin wax sections and better than that achieved when removing areas from paraffin wax blocks, but with a degree of cellular preservation similar to that of glutaraldehyde fixed tissue.

Rapid processing can be adapted because of section thickness, thus enabling electron microscopy to be carried out within two hours of receipt.

The cryostat section used in this way has considerable potential. Areas worth exploring include immunocytochemistry and enzyme histochemistry, where standard methods could be performed on the glutaraldehyde-fixed sections before processing through to resin for electron microscopy. Labelling procedures would require modification so that sufficiently electron dense labels or final reaction products were used. With regard to enzyme histochemistry work has been carried out ${ }^{10}$ localising lysosomal and non-lysosomal acid phosphatase at electron microscopical level by using the cryostat section, but this was found inferior to equivalent work using vibratome sections of nervous tissue. Further investigation is warranted in view of the far superior localisation afforded by the cryostat section.
Another instance where this method could prove valuable is in the cytological study of fluid aspirates. By cutting cryostat sections of artificially-clotted fluid aspirates ${ }^{11}$ routinely, a source of material would be available for electron microscopical studies if a situation occurred where light microscopy gave an equivocal diagnosis of a poorly differentiated tumour. Although there are a number of other techniques available to aid correlation of cytological material between light and electron microscopical levels-for example, split samples, stereoscopic examination of fixed or unfixed tissues, mirror imaging techniques-the technique described here guarantees that the tissue sample examined is exactly the same for light microscopy as it is for electron microscopy.

In summary this technique may permit a number of different histological investigations to be carried out on a source of tissue where both light and electron microscopical evaluation may be necessary.

The authors would like to thank the members of staff in the Pathology Laboratory at Weston Park Hospital for their help and critical assessment of the manuscript, Mrs JA Griffiths for her patience and typing of the manuscript and Mrs $J$ Rhodes for her photographic expertise.

\section{References}

${ }^{1}$ Rowden G, Lewis MG. Experience with a three hour electron microscopy service. J Clin Pathol 1974;27:505-7.

${ }^{2}$ Luft JH. Improvements in epoxy resin embedding. $J$ Biophys Biochem Cytol 1961;9:409-14.

${ }^{3}$ Gonzalez-Angulo A, Ruiz de Chavez I, Castaneda M. A reliable method for electron microscopical examination. Am J Clin Pathol 1978;70:697-9.

4 Sabatini DD, Bensch K, Barrnett RJ. Preservation of cellular ultrastructure and enzyme activity and aldehyde fixation. $J$ Cell Biol 1963;17:19-58.

${ }^{3}$ Robinson G. In: Bancroft JD, Stevens A, eds. Theory and practice of histological technique. London: Churchill-Livingstone, 1982:497.

- Carr I, Toner PG. Rapid electron microscopy in oncology.J Clin Pathol 1977;30:13-5.

' Johannessen JV. Rapid processing of kidney biopsies for electron microscopy. Kidney Int 1973;3:46-50.

${ }^{8}$ Sokol RJ, Norris PD, Hudson G. Examination of skin window preparations by transmission electron microscopy. J Clin Pathol 1980;33:697-8.

- Reynolds ES. The use of lead citrate at high pH as an electron opaque stain in electron microscopy. J Cell Biol 1963;17:208-12.

${ }^{10}$ Wilks PN. An optimized lead capture electron histochemical technique for the demonstration of lysosomal and nonlysosomal acid phosphatase activity in nervous tissue. Med Lab Sci 1980;37:149-64.

"Gregory PA. Cell block preparation: plasma-thrombin technique. Histo-Logic 1981;XI:165.

Requests for reprints to: Mr P Norris, Department of Pathology, Weston Park Hospital, Sheffield S10 2SJ, England. 